\title{
Standardization of Laparoscopic Left Hemihepatectomy by Extrahepatic Glissonean Pedicle Isolation and HV Root - At First One-way Resection Based on Laennec's Capsule
}

\author{
Yutaro Kato, Atsushi Sugioka, Yoshinao Tanahashi, Gozo Kiguchi, Masayuki Kojima, \\ Sanae Nakajima, Akira Yasuda, Jun-ichi Yoshikawa, Ichiro Uyama
}

Corresponding author:

Yutaro Kato, MD

Department of Surgery

Fujita Health University

1-98, Dengakugakubo

Kutsukake-cho, Toyoake

Aichi, 470-1192, Japan

Tel: $+85-562-93-9254$

Fax: +85-562-93-7060

E-mail: y-kato@fujita-hu.ac.jp
Department of Surgery, Fujita Health University, Toyoake, Aichi, Japan

\begin{abstract}
Laparoscopic left hemihepatectomy (LLH) is one of the major hepatectomy procedures and its surgical techniques are not well standardized. According to our surgical strategies for anatomic liver resections, which are composed of the extrahepatic Glissonean pedicle approach (GPA) and hepatic vein (HV) root-at first one-way parenchymal dissection, we have standardized the surgical techniques for LLH. Both approaches are based on the anatomical background of Laennec's capsule. The right and left sides of the root of the Glissonean pedicle including the umbilical portion of the portal vein (G-UP) are dissected with Laennec's capsule preserved on the liver parenchyma. The G-UP is isolated extrahepatically and divided using a stapler. The common trunk of the left hepatic vein (LHV) and middle hepatic vein (MHV) is exposed from the left cranio-dorsal side and LHV is divided at its root. Parenchymal dissection starts from exposing the root of MHV, which is tracked from the root side to the peripheral side in one direction. Left hemihepatectomy is completed at the left side of the gall bladder bed. In conclusion, LLH can be standardized by initial isolation and division of G-UP according to the Laennec's capsule-based extrahepatic GPA followed by HV root-at first cranio-caudal, one-way parenchymal resection.
\end{abstract}

Key words: left hemihepatectomy, laparoscopic liver resection, Glissonean pedicle, Laennec's capsule, plate system, hepatic vein

\section{INTRODUCTION}

Left hemihepatectomy is one of the major hepatectomy procedures and standardization of its surgical techniques is of importance for patient safety and increased oncologic curability. Laparoscopic left hemihepatectomy (LLH) has increasingly become a standard practice alternative to open approach in the experts' hands (1), but its surgical techniques vary widely among surgeons and no standardization has been reached. The methods of inflow control for LLH are largely classified into two approaches: the controlled method with intrafascial hilar dissection of the individual vessels, and the Glissonean pedicle approach (GPA) (1-4). The GPA to the Glissonean pedicle including the umbilical portion of 
the portal vein (G-UP) includes both intrahepatic (5)) and extrahepatic $(3,4)$ techniques. On the other hand, approaches to the common trunk of the left hepatic vein (LHV) and middle hepatic vein (MHV) as well as parenchymal dissection in LLH include those by ventral and caudal techniques $(1,4)$. Previous reports have shown acceptable short-term surgical results with low morbidity and mortality in $\operatorname{LLH}(1,3,4)$.

Our basic approaches to anatomic liver resections are composed of two techniques: the extrahepatic GPA and hepatic vein (HV)-root at first cranio-caudal, oneway parenchymal resection, regardless of the types of hepatectomy. Both techniques are based on the anatomical background of Laennec's capsule (6).

In this article with a video presentation, we describe our standardized techniques for LLH and discuss its features and potential merits and limitations.

\section{SURGICAL TECHNIQUES}

The patient is placed at the supine position with the legs apart. The operating surgeon stands at the left side of the patient and the scope operator between the legs. The optical port is placed at the umbilicus and the pneumoperitoneal pressure is set at $10 \mathrm{mmHg}$. Laparoscopy is performed with a flexible scope. The operational $12-\mathrm{mm}$ ports are placed at the right lateral subcostal region, right mid-clavicular subcostal region and the left mid-clavicular subcostal region. Two additional 5-mm ports are placed at the right and left mid-abdomen on the parasternal line, respectively. The round ligament is divided, and its liver-side stump is lifted up to the epigastrium and fixed there to obtain a wide surgical field of the hepatic hilum.

The first step is the dissection of G-UP. According to the Gate theory proposed by Sugioka et al. (6), the right side of the root of G-UP is assigned to Gate III and the left side to Gate I. To dissect Gate III, the left part of the hilar plate is firstly detached from the Laennec's capsule of the confronting parenchyma of the quadrate lobe. After incising the reflecting serosa at the border between the hepatoduodenal ligament and the liver surface, you have to divide one or two pieces of membranous or cord-like tissue connecting the anterior liver surface and the hepatoduodenal ligament behind the serosa. We call this tissue 'the anchor'. Only by dividing 'the anchors,' you can enter the space between the hilar plate and the contiguous Laennec's capsule of the confronting liver parenchyma. By dissecting the left part of the hilar plate and part of the right side of the umbilical plate, you can dissect Gate III and the cranial space behind the umbilical plate of G-UP from the right side without parenchymal dissection, for which the right-sided view obtained by the flexible scope is useful. Next, Gate I is dissected by approaching the corner formed by the left side of GUP and the hilar root of the Arantius plate (ArP). With the aid of the left-sided view created by the flexible scope, the cranial space behind the root of the umbilical plate above ArP is dissected from the left side. By connecting the dissecting planes from the right and left sides of G-UP, you can pass a tape behind G-UP (usually from Gate III to Gate I) and isolate it without parenchymal dissection. Then, G-UP is divided using a $60-\mathrm{mm}$ linear stapler inserted from the right lateral subcostal port, with a special attention not to compromise the hilar plate or ArP. The demarcation line on the Rex-Cantlie line is confirmed and marked.

The second step is the dissection of the common trunk of LHV and MHV. The supra-hepatic pre-caval fascia and the anterior surface of the common trunk are dissected. Then, the left lateral section (LLS) is mobilized and flipped up to the right side over the segment IV of the liver. A good operative field can be obtained by the left latero-dorsal view. The serosal reflection between the segment II and ArP is incised and by dissecting around the attachment of ArP to LHV or inferior vena cava (IVC), you can expose the root of LHV and the anterior surface of IVC from the laterodorsal side. Dissecting the LHV from the dorsal side facilitates its extrahepatic isolation in most cases. The isolated LHV is divided and the left liver is overturned to the orthotopic position.

The third step is parenchymal dissection. It starts from exposing the root of MHV and continues from the root side to the peripheral side along MHV in the cranio-caudal direction. Several hepatic venous tributaries from the segment IV joining MHV are serially divided. We call such method of liver transection as 'the HV root-at first one-way resection'. On dissecting and exposing the MHV, it is important to recognize Laennec's capsule on the wall of MHV. During cranio-caudal dissection of the major HVs from their roots, particularly by an ultrasonic aspirator, Laennec's capsule is naturally attached to or preserved on the wall of MHV (6). The parenchymal dissection finishes just on the left side of the gall bladder, which is not removed in our usual manner of left hemihepatectomy, and LLH is completed. We normally do not use Pringle maneuver during LLH. The specimen is extracted from the extended umbilical incision. 


\section{DISCUSSION}

Although left hemihepatectomy is one of the major liver resection procedures, we think that it would be suitable for technical standardization, because of the technical simplicity of inflow and outflow control and of the straightforward parenchymal cutting line. To standardize any types of anatomic liver resections, we have focused on the anatomical relevance of Laennec's capsule to the practical techniques (6). Our basic policies for anatomic liver resections are the inflow control by the extrahepatic GPA and the outflow control and parenchymal dissection by the HV-root at first craniocaudal, one-way parenchymal dissection. Furthermore, laparoscopic magnified and caudal view reinforces precise understanding of the anatomical structures. Combining our surgical strategies in anatomic liver resections and the advantages of laparoscopic approach, we have standardized surgical techniques for LLH based on Laennec's capsule as presented here. Patient safety was preserved in our experience with consecutive 13 LLH cases, where the median blood loss was $125 \mathrm{~g}$ and no complications $\geq$ Clavien-Dindo Grade II were noted (unpublished data).

Several methods of pedicle control and those of approaches to the common trunk of LHV and MHV have been reported on LLH (1). There seem to be three methods to control the left portal pedicle: hilar intrafascial dissection of the individual vessels to the left liver, intrahepatic or extrahepatic GPA (2). In the intrafascial approach during LLH, the left portal vein and hepatic artery are mostly divided within the hilar plate tissue, and so it is a norm that several branches to the left caudate lobe are sacrificed. Moreover, as the dividing line for the left bile duct is set at the peripheral side of the hilar plate, the cutting level is a slightly different between the vascular structures and the bile duct. In contrast, in the pedicle control method by GPA, either intrahepatic or extrahepatic, all the portal triad elements inside G-UP are divided en bloc at the peripheral side of the hilar plate, which serves to avoid compromising other parts of the liver than the left liver. From the technical simplicity and procedural safety, we prefer GPA as a standard procedure of hilar pedicle isolation in LLH.

In either open or laparoscopic approach, we prefer to isolate G-UP according to the 'extrahepatic' GPA, for which the utilization of Laennec's capsule and the Gate theory (6) is essential and may confer potential advantages particularly in LLH. By using this approach, in which you dissect the layer between the Glissonean pedicle sheath and Laennec's capsule of the confronting parenchyma at the right access points (Gates I and III), you can avoid unpleasant bleeding due to the damage of parenchyma or MHV nearby. A clear exposure of the surface of the hilar plate, G-UP and ArP allows us to determine the safe line of G-UP division. Furthermore, the laparoscopic magnified and multiangled view is helpful for identifying the accurate dissection layer behind G-UP, which may be hard to dissect through a narrow space in the open setting. In addition, early division of G-UP before parenchymal dissection may provide two advantages. One is that the division (or clamping) of G-UP is mandatory before severance of LHV to avoid hepatic congestion during parenchymal dissection. The early division of LHV is helpful for opening the liver resection plane from the cranial side. The other merit is that division of G-UP increases mobility of the left liver. It further facilitates the rightward overturn of LLS, by which the dorsal surgical field around the HV common trunk becomes wide and clear, particularly in our method of LLH. Together with the early liberation of LLS by dividing LHV, the increased mobility of LLS further provides effective caudal traction and handling of the left liver during the cranio-caudal liver transection.

Besides its importance in the extrahepatic GPA, Laennec's capsule has an anatomical implication to the technical aspects on the exposure of the major HV walls. Our experience suggests that during craniocaudal liver resection along the MHV trunk, the dissection naturally enters the layer between the parenchyma and Laennec's capsule (outer-Laennec approach). Otherwise, you can dissect the layer between the Laennec's capsule (hepatic Laennec's capsule) and the MHV wall, on which another capsule, the cardiac Laennec's capsule as we have named, is potentially preserved (inter-Laennec approach) (7). In our method of LLH, during exposure of the roots of MHV and LHV, the inter-Laennec layer can be easily entered from the dorsal side of the MHV after mobilization of LLS and division of LHV. Furthermore, in our experience, dissection of the inter-Laennec layer is easier than that of the outer-Laennec's layer, because the attachment between the hepatic and cardiac Laennec's capsules is usually looser than that between the liver parenchyma and hepatic Laennec's capsule. Additionally, because in the inter-Laennec approach, the debris of liver parenchyma should not remain on the MHV wall as in the outer-Laennec approach, fine anatomy of MHV tributaries can be easily followed. Therefore, we consider that during exposure of MHV and cranio-caudal parenchymal dissection in LLH, the inter-Laennec approach is more useful than the 
outer-Laennec's approach. However, regardless of the approaches, it is of utmost importance to recognize accurately the layers being dissected and to select the optimal approach depending on the condition of the vein wall, bleeding status and feasibility and safety of dissecting the layers. Laparoscopic magnified view is sure to help recognize precisely such minute anatomical structures and dissection layers.

There may be several advantages in the HV-root at first cranio-caudal one-way parenchymal resection in LLH. First, each root of LHV and MHV is securely and safely exposed, by which the potential anatomical misjudgment is reduced and the venous tributaries of $\mathrm{MHV}$ can be divided at their roots without concerns of wrong severance. Second, because the MHV tributaries join the trunk at an obtuse angle in the cranial view, sweeping the parenchymal tissue along the MHV trunk in the cranio-caudal direction prevents tearing or splitting of the tributaries. Third, as the liver is gradually opened from the left cranial side, even if a critical bleed occurs near the root of MHV, it can be safely controlled in a good operative view. Fourth, as mentioned above, Laennec's capsule is naturally preserved on the exposed wall of MHV during the cranio-caudal resection, and therefore, the vein wall is maintained thick and strong. Even a bleed on the MHV trunk, unless it is massive, is mostly controllable by a simple touching coagulation, probably due to the coagulation-induced contraction of the Laennec's capsule fibers on the vein, which serves to close the bleeding hole.

Our method of LLH may have several technical and oncological limitations. Technical limitations to the extrahepatic GPA are largely related to the quality of the liver parenchyma. In cases with underlying fatty liver, the extrahepatic GPA, which preserves Laennec's capsule on the liver side, is sometimes technically difficult, because the capsule is very fragile and easy to rupture. Tumors located near the hepatic hilum or the hepatic vein root could be excluded from the indication of our approach, because it may compromise the surgical margin. However, the extrahepatic GPA and the exposure of the HV root with one-way parenchymal resection could be applied to tumors compressing the hilar plate or the HV root in selected cases where they are fully capsulized without direct invasion to such structures.

\section{CONCLUSION}

In summary, LLH can be safely standardized by extrahepatic GPA and HV-root at first cranio-caudal one-way parenchymal resection based on Laennec's capsule.

\section{Disclosures}

The authors have no conflicts of interest to disclose relevant to the content of this article.

\section{Author's contributions}

YK and AS equally contributed to this study

\section{REFERENCES}

1. Valente R, Sutcliffe R, Levesque E, Costa M, de' Angelis N, Tayar C, et al. Fully laparoscopic left hepatectomy - a technical reference proposed for standard practice compared to the open approach: a retrospective propensity score model. HPB (Oxford). 2018; 20 (4):347-355.

2. Couinaud C: Surgical Anatomy of the Liver Rvisited. Paris: pers Ed; 1989

3. Cho A, Yamamoto $\mathrm{H}$, Kainuma O, Ota T, Park S, Yanagibashi $\mathrm{H}$, et al. Arantius ligament approach for the left extrahepatic Glissonean pedicle in pure laparoscopic left hemihepatectomy. Asian J Endosc Surg. 2012; 5 (4):187-190

4. Okuda Y, Honda G, Kurata M, Kobayashi S, Sakamoto K. Dorsal approach to the middle hepatic vein in left hemihepatectomy. J Am Coll Surg. 2014; 219 (2): e1-e4.

5. Machado MAC, Makdissi FF, Herman P, Surjan RC. Intrahepatic Glissonian approach for pure laparoscopic left hemihepatectomy. J Laparoendosc Adv Surg Tech A. 2010; 20 (2):141-142.

6. Sugioka A, Kato Y, Tanahashi Y. Systematic extrahepatic Glissonean pedicle isolation for anatomical liver resection based on Laennec's capsule: proposal of a novel comprehensive surgical anatomy of the liver. J Hepatobiliary Pancreat Sci. 2017; 24 (1):17-23.

7. Kiguchi $G$, Sugioka A, Kato $Y$, Uyama I. Use of the inter-Laennec approach for laparoscopic anatomical right posterior sectionectomy in semi-prone position. Surg Oncol. 2019; 29:140-141. 